\title{
Analysis and Dimensioning of a Single-Layer Optical Network Based on a "Switchless" Concept in Relevant Scenarios
}

\author{
S. Binetti *, A. Bosco *, M. Listanti ${ }^{\S}$, A. Maga *, R. Sabella ${ }^{\wedge}$ \\ ${ }^{*}$ CoRiTeL - Consorzio di Ricerca sulle Telecomunicazioni, Roma, Italy; E-mail: \\ bosco@coritel.it; fax +396 72597439; tel. +396 72597441; Via di Tor Vergata 110, 00133, \\ Rome, Italy. \\ \$INFOCOM Dept - Università di Roma "La Sapienza", Rome, Italy; E-mail: \\ marco@infocom.ing.uniromal.it; fax +396 48906114; tel. +396 44585640; Via Eudossiana \\ 18, 00184, Rome, Italy. \\ ${ }^{\wedge}$ CoRiTeL - Ericsson Telecomunicazioni, Research \& Develop. Division, Rome, Italy; \\ Corresponding author: Roberto Sabella; E_mail: sabella@coritel.it; fax +39 06 72597439; tel. \\ +39 6 72597442; Via Anagnina 203, 00040, Roma, Italy.
}

Key words: Switchless, WDM, WDMA/TDMA, network analysis and dimensioning, transport network architecture, wavelength converter.

Abstract: The "switchless" all-optical network is an alternative networking approach, developed in the framework of the ACTS project named SONATA (Switchless Optical Network for Advanced Transport Network), which aims to provide a future single-layer, advanced transport architecture on a national scale. The single-hop, shared-access network employs time and wavelength agility, using fast tunable transmitters and receivers. The dimensioning of this type of network is one of the main tasks for the design of the network, which serves a certain number of customers, connected together by means of Passive Optical Networks (PONs). This paper reports an analytical model which allows network dimensioning, according to relevant design parameters: number of customers per PON, number of PONs, offered traffic per single user (residential or business), number of total wavelength converters, and the required system performance expressed in terms of blocking probability. 


\section{INTRODUCTION}

The "switchless" concept was born as an alternative transport network architecture; it has been proposed with the aims of avoiding the need of large and fast switching nodes, simplifying network structure and layer architecture within the network [1], [2]. The proposal consists of a singlelayer network platform for end-to-end optical connections, able to serve a large number of terminals, distributed on a wide geographical area, and to handle residential and business traffic. This network model offers a multipleaccess, based on a single node providing only passive routing functions [3] [7]: it is realised by a WDMA/TDMA scheme [1], [2]. On this basis, a terminal is connected to the Passive Wavelength Routing Node (PWRN) by means of a set of wavelengths, each one carrying a TDM flow. In Figure 1, it is shown the system scheme.

The structure is formed by $\mathrm{N}_{\mathrm{p}}$ PONs, $\mathrm{N}_{\mathrm{t}}$ fast tunable terminals (belonging to each PON), $\mathrm{N}_{d}$ re-circulating lines (or "dummy ports"), and $\mathrm{N}_{\mathrm{c}}$ lines dedicated to signalling. In this way, the total number of PWRN ports is $\mathrm{N}=\mathrm{N}_{\mathrm{p}}+\mathrm{N}_{\mathrm{d}}+\mathrm{N}_{\mathrm{c}}$. In general, cascade optical amplifiers are required, and, in large-split "HyperPONs", a noise gating functional block has to be introduced after those amplifiers for filtering out-band noise [10] [11]. As stated above about the access, a direct connection between two terminals, belonging to the same or to different PONs, is set up by the network control selecting a time-slot on that particular wavelength, which is associated to a specific input PON. This way of working allows us to define the "switchless" concept in the sense that physical switching function is removed from the network, limiting it to the terminals (infact, neither electronic switches/cross-connects [8], nor optical cross-connects [9] are needed). Of course, a centralized network control is required, but architecture simplifications and hardware reductions are obtained; moreover, collisions among calls are prevented by a cyclic assignment of wavelengths at the input of PWRN and by TDMA scheme. In Table 1, it is reported an example of wavelength assignment. To achieve a higher degree of flexibility in the use of network capacity and to support traffic that cannot be served directly (PON-to-PON connection), a set of $\mathrm{N}_{d}$ wavelength converter arrays, on recirculating lines, is introduced (they offer auxiliary capacity to PWRN). In this way, a variable number of wavelengths, depending on traffic requirements, can be dynamically allocated between any pair of terminals: if a direct connection is not available, a call will be routed from a source PON towards the destination PON via a proper wavelength conversion, by using a free time-slot on a wavelength connecting the source PON with a "dummy port", and the same time-slot on the wavelength connecting that "dummy port" to the destination PON. 
In $\S 1$, it has been presented the "switchless" network scheme; in $\S 2$, it will be presented the analytical model, with network dimensioning considering a telephone traffic uniformly distributed (\$2.1), and a telephone traffic with unbalanced distribution and limitation of wavelength converters number ( $\$ 2.2)$; in $\S 3$, conclusions will be presented.

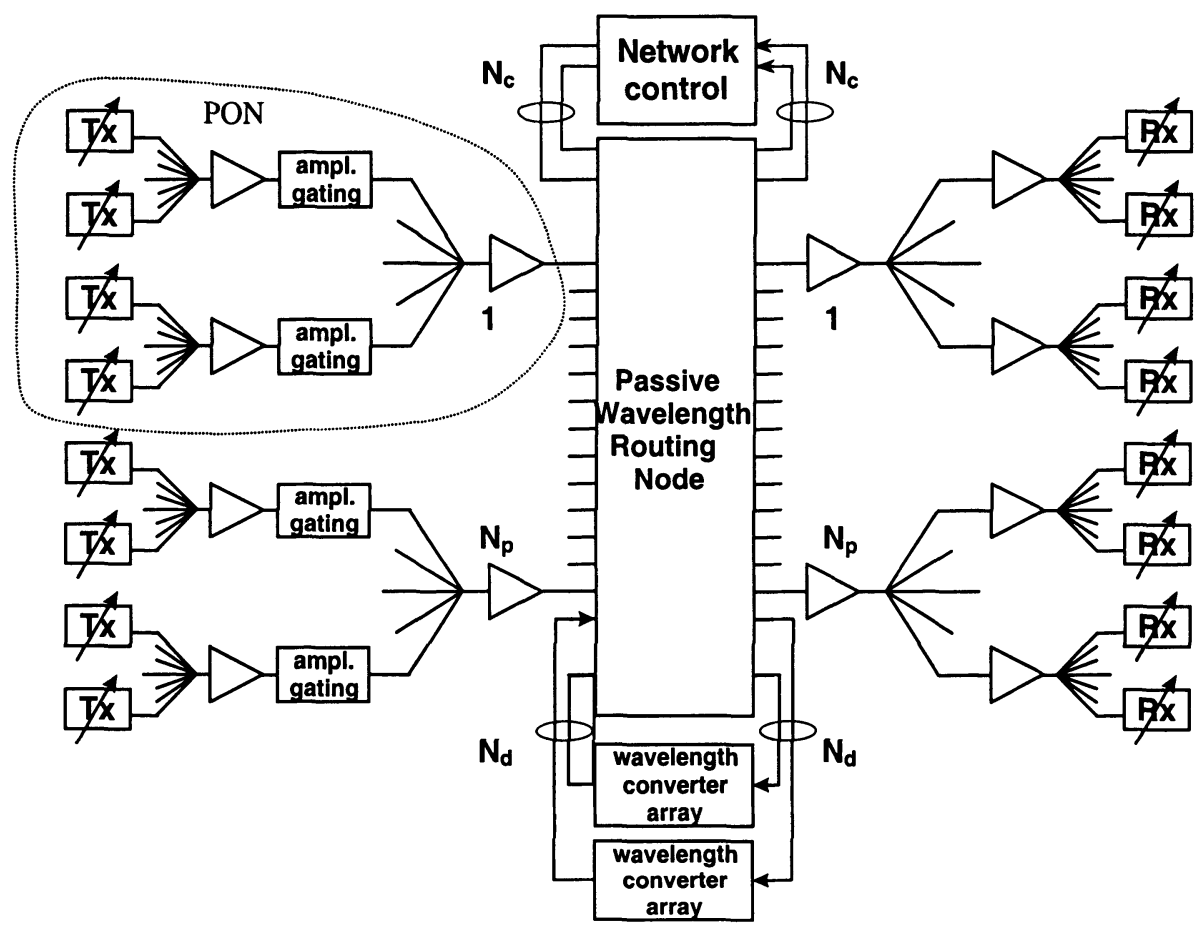

Figure 1. Complete "switchless" network structure

Table 1. Example of wavelengths conflict free allocation in $4 \times 4$ PWRN with $\mathrm{Np}=4$ and $\mathrm{Nd}=2$

\begin{tabular}{|c|c|c|c|c|c|c|c|}
\hline & \multicolumn{4}{|c|}{$\mathrm{N}_{\mathrm{p}}$ ports } & \multicolumn{2}{|c|}{$N_{d}$ ports } \\
\hline & & 1 & 2 & 3 & 4 & 5 & 6 \\
\hline \multirow{4}{*}{$\begin{array}{l}\stackrel{0}{0} \\
\vdots \\
z^{2}\end{array}$} & 1 & 1 & 2 & 3 & 4 & 5 & 6 \\
\hline & 2 & 6 & 1 & 2 & 3 & 4 & 5 \\
\hline & 3 & 5 & 6 & 1 & 2 & 3 & 4 \\
\hline & 4 & 4 & 5 & 6 & 1 & 2 & 3 \\
\hline \multirow{2}{*}{$z_{0}$} & 5 & 3 & 4 & 5 & 6 & 1 & 2 \\
\hline & 6 & 2 & 3 & 4 & 5 & 6 & 1 \\
\hline
\end{tabular}




\section{MODELLING FOR NETWORK DIMENSIONING}

To assess the feasibility of the "switchless" network concept, it is necessary to investigate how the network should be dimensioned in terms of available resources, considering relevant network scenarios and realistic traffic distributions. In the framework of the ACTS-SONATA project, three relevant cases have been studied: small, medium, and large scale networks, referring to residential and business customers. In the analysis, it has been considered telephone traffic, both with uniform and unbalanced distribution. The choice of telephone traffic allows both the utilisation of Poisson traffic model and the achievement of meaningful results, so highlighting the main system properties.

\subsection{Analytical model - uniform traffic distribution}

We assume to have uniform traffic distribution between input and output PONs. In order to investigate the network performance, the blocking probability has been evaluated both as a function of network resources (number of PONs, number of "dummy ports", number of wavelength converters per "dummy port") and network requests (number of users). In this sense, the network dimensioning can be accomplished by evaluating network parameters that guarantee a blocking probability less than a fixed value, which represents a network quality parameter (e.g. $\left.10^{-5}\right)$. An exact computation of the blocking probability would require a solution of an $\mathrm{N}_{\mathrm{p}}$ dimensional Markov chain, with a states number equal to $\mathrm{T}^{\mathrm{Np}}$ : such a model becomes too complex already with few PONs and a little time-slots number (T).

To overcome this problem, it has been analysed a single traffic relation between the i-th input PON and the j-th output PON, and it has been evaluated the corresponding blocking probability taking into account traffic effects, related to the other traffic relations. According to uniform traffic hypothesis, this probability is also the blocking probability associated with the whole system. The logical model is shown in Figure 2. In Table 2, the notations used are defined. 


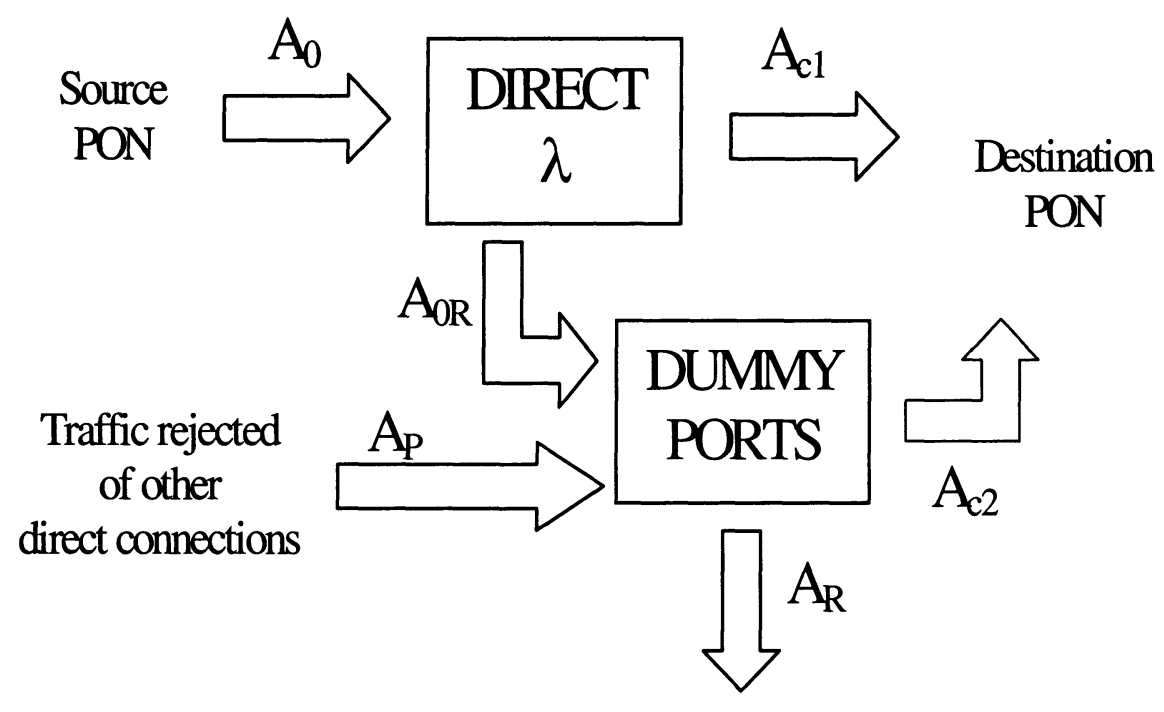

Figure 2. Scheme of the analytical model

In order to analyse the model sketched in Figure 2, the following simplifying hypotheses have been done:

- wavelengths connecting the source PON to the "dummy ports" are considered to be blocking free. This means that input PONs have a dedicated wavelength converter per "dummy port", in order to establish connection input PON-output PON through a suitable wavelength conversion at the output of a "dummy port". This assumption allows the evaluation of the blocking probability due to the "dummy ports", as exclusively depending on the saturation of time-slots of a specific wavelength connecting the "dummy ports" with the destination PONs.

- traffic refused by direct wavelengths and offered to the "dummy ports" $\left(A_{o, R}, A_{P}\right)$ is represented by only its first two moments (the mean and the variance); this assumption allows the application of the Equivalent Random Traffic (ERT) method [12] for the analysis of the blocking probability of the wavelength outgoing from the "dummy ports".

Four steps support the analysis:

1. evaluation of the loss probability $\left(\pi_{1}\right)$ experienced on the direct wavelengths (PON-PON): this is the probability that a generic call can not be handled by direct service, and it must go towards "dummy ports";

2 . evaluation of the traffic refused by "dummy ports" and calculation of the relative blocking probability $\left(\pi_{2}\right)$; 
3. calculation of the total loss probability $(\pi)$ : this is the probability that a generic call cannot be accommodated anyway.

Table 2. Notations for the network parameters used

\begin{tabular}{|c|c|}
\hline Parameter & Definition \\
\hline $\mathrm{A}_{\mathrm{o}}$ & $\begin{array}{l}\text { Average traffic intensity offered for single relation } \\
\text { input/output; }\end{array}$ \\
\hline$A_{0, t o t}$ & Average traffic intensity offered globally $\left(\mathrm{A}_{\mathrm{o}, \mathrm{tot}}=\mathrm{N}_{\mathrm{p}} * \mathrm{~A}_{\mathrm{o}}\right)$; \\
\hline $\mathrm{A}_{\mathrm{cl}}$ & $\begin{array}{l}\text { Average traffic carried by a direct wavelength between } \\
\text { an input and an output PON; }\end{array}$ \\
\hline$A_{o, R}$ & $\begin{array}{l}\text { Average traffic rejected for single relation input/output } \\
\text { and offered to the "dummy ports"; }\end{array}$ \\
\hline$A_{P}$ & $\begin{array}{l}\text { Average traffic rejected by all direct wavelengths and } \\
\text { offered to the "dummy ports" }\left(A_{P}=\left(N_{p}-1\right)^{*} A_{O, R}\right)\end{array}$ \\
\hline $\mathrm{A}_{\mathrm{c} 2}$ & Average traffic carried globally by the "dummy ports"; \\
\hline$A_{R}$ & Average traffic rejected by the system; \\
\hline
\end{tabular}

On the basis of what said until now, we have three different blocking probabilities: the first at the input of direct service (input of router ports), the second at the input of "dummy ports", the third at the output of "dummy ports". In order to calculate the first loss probability, considering that direct wavelengths connecting the source and the destination PONs are considered as a group of $\mathrm{T}$ servers with at the input a Poisson traffic of average value $A_{o}$, it has been applied the Erlang-B analytical model; thanks to that, parameters as $\pi_{1}(1), A_{o, R}(2)$, standard deviation $\sigma_{o, R}^{2}(3)$, and the total traffic refused (4) by direct service can be calculated:

$$
\begin{aligned}
& \pi_{1}=B\left(A_{o}, T\right)=\frac{A_{o}^{T} / T !}{\sum_{j=o}^{T} A_{o}^{j} / j !} \\
& A_{o, R}=A_{o} \cdot \pi_{1}
\end{aligned}
$$




$$
\begin{aligned}
& \sigma_{o, R}^{2}=A_{o, R} \cdot\left(1-A_{o, R}+\frac{A_{o}}{T+1-A_{o}+A_{o, R}}\right) \\
& A_{o, \text { dummy }}=A_{o, R}+A_{P}=A_{o, R}+\left(N_{P}-1\right) \cdot A_{o, R}=N_{P} \cdot A_{o, R}
\end{aligned}
$$

Similarly, in order to calculate the blocking probability at the input of "dummy ports", traffic rejected by "dummy ports" must be calculated. This traffic is known to be peaked: so, it cannot be applied the Erlang model. However, according to the ERT method, this traffic can be thought as the overflow traffic of a group of $\mathrm{N}^{*}$ servers (called preamble system) loaded by Poisson traffic of average value $\mathrm{A}^{*}$. These equivalent parameters can be calculated, solving the following two-equation system (5):

$$
\left\{\begin{array}{l}
A_{o, \text { dummy }}=A^{*} \cdot B\left(A^{*}, N^{*}\right) \\
V_{o, \text { dummy }}=A_{o, \text { dummy }} \cdot\left(1-A_{o, \text { dummy }}+\frac{A^{*}}{N^{*}+1-A^{*}+A_{o, \text { dummy }}}\right)
\end{array}\right.
$$

Once $\mathrm{A}^{*}$ and $\mathrm{N}^{*}$ are known, the mean value of the traffic overflowing the "dummy port" system (6) and the blocking probability at the input of "dummy ports" (7) are given by:

$$
\begin{aligned}
& A_{R}=A^{*} \cdot B\left[A^{*},\left(N^{*}+N_{d} \cdot T\right)\right] \\
& \pi_{\text {in }}={ }^{A} / A_{o, \text { dummy }}
\end{aligned}
$$

Now, to complete the analysis and to find the total blocking probability, we must take into account conflicts at the output of the "dummy ports": to do this, "dummy ports" have been considered as a cascade of two gates, in which offered traffic can be rejected by the second block. In this case the evaluation of the second loss probability is given by:

$$
\pi_{2}=1-\left(1-\pi_{\text {in }}\right) \cdot\left(1-\pi_{\text {out }}\right)
$$


where:

- $\left(1-\pi_{\text {in }}\right)$ is the probability that there is at least one slot for access to recirculating line, considering traffic rejected by direct connections;

- $\left(1-\pi_{\text {out }}\right)$ is the probability that there is at least one slot to leave the recirculating line, considering only the traffic that has passed the input block of "dummy ports".

The blocking probability at the output of the "dummy ports" is calculated by Frederick's model.

To be noticed that this model is not rigorous: it doesn't take into account the coincidence of time-slots at the input and output of "dummy ports". The total blocking probability is given by $\pi=\pi_{1} * \pi_{2}$.

\subsubsection{Network dimensioning results for uniform traffic distribution}

To investigate system performance, we consider three different scenarios (large, medium, small region), corresponding to different sizes of a region served by the system, and two user classes (residential and business); the data used for the study are reported in Table 3. A single business user presents a traffic intensity of 0.15 Erl, while a residential user presents a traffic intensity of $0.05 \mathrm{Erl}$; the average call holding time is supposed to be equal for both classes. The scenarios introduced are analysed considering a) full residential ( $100 \%$ of residential users); b) mixed (78\% of residential users and $22 \%$ of business users); c) full business (100\% of business users). Finally, $T=1000$ is the maximum number of time-slots of the TDM flow dedicated to the telephone traffic on each wavelength line accessing the PWRN.

Table 3. Network and traffic scenarios for system performance analysis

\begin{tabular}{cc|ccc}
\hline \multicolumn{2}{c|}{ Network scenarios } & \multicolumn{3}{c}{ Traffic scenarios } \\
\hline Type & $\begin{array}{c}\text { Number of } \\
\text { users }\end{array}$ & Residential & Mixed & Business \\
Small region & 15000 & $750 \mathrm{Erl}$ & $1080 \mathrm{Erl}$ & $2250 \mathrm{Erl}$ \\
Medium region & 150000 & $7500 \mathrm{Erl}$ & $10800 \mathrm{Erl}$ & $22500 \mathrm{Erl}$ \\
Large region & 2000000 & $100000 \mathrm{Erl}$ & $144000 \mathrm{Erl}$ & $300000 \mathrm{Erl}$ \\
\hline
\end{tabular}

In Figure 3, for instance, are reported the values of the PWRN port capacity, $\mathrm{C}_{\mathrm{s}}\left(\mathrm{N}_{\mathrm{p}}\right)$, expressed in number of time-slots vs. the number of PONs, for the different considered scenarios. To be noticed that $\mathrm{C}_{s}\left(\mathrm{~N}_{\mathrm{p}}\right)$ is defined as the minimum between 1000 time-slots and the minimum value to guarantee the $1^{\text {th }}$ loss probability (only for direct service) less than 0.1 . From this 
figure, we can see that increasing the number of PONs, $\mathrm{C}_{\mathrm{s}}\left(\mathrm{N}_{\mathrm{p}}\right)$ decreases because the total offered traffic per PWRN port decreases. Moreover, it is intuitive to understand that, for a given loss probability and PWRN capacity, increasing the number of PONs, the number of "dummy ports" (or, equivalently, the auxiliary capacity on re-circulating lines) decreases, because calls, on average, can be handled through direct connections

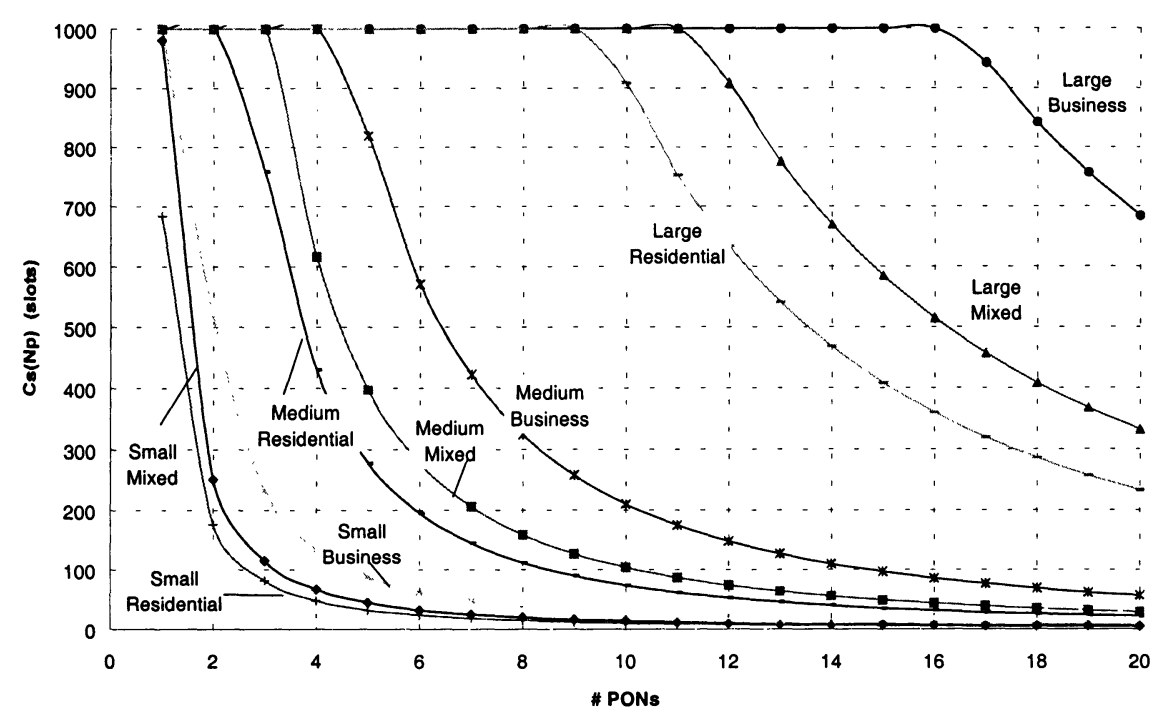

Figure 3. Number of slots vs. number of PONs in considered system scenarios

A parameter that can be chosen for representing the system cost is the total number of ports $\left(\mathrm{N}_{\mathrm{t}}\right)$ of the PWRN. In Figure 4, it is sketched the total number of ports vs. the number of PONs $\left(\mathrm{N}_{\mathrm{d}}\right)$ :

- for low $\mathrm{N}_{d}$ values, the PWRN size decreases as the number of "dummy ports" increases;

- for higher values of the number of "dummy ports", PWRN size linearly increases with $\mathrm{N}_{\mathrm{d}}$.

The threshold between the two zones is the best choice for system configuration. 


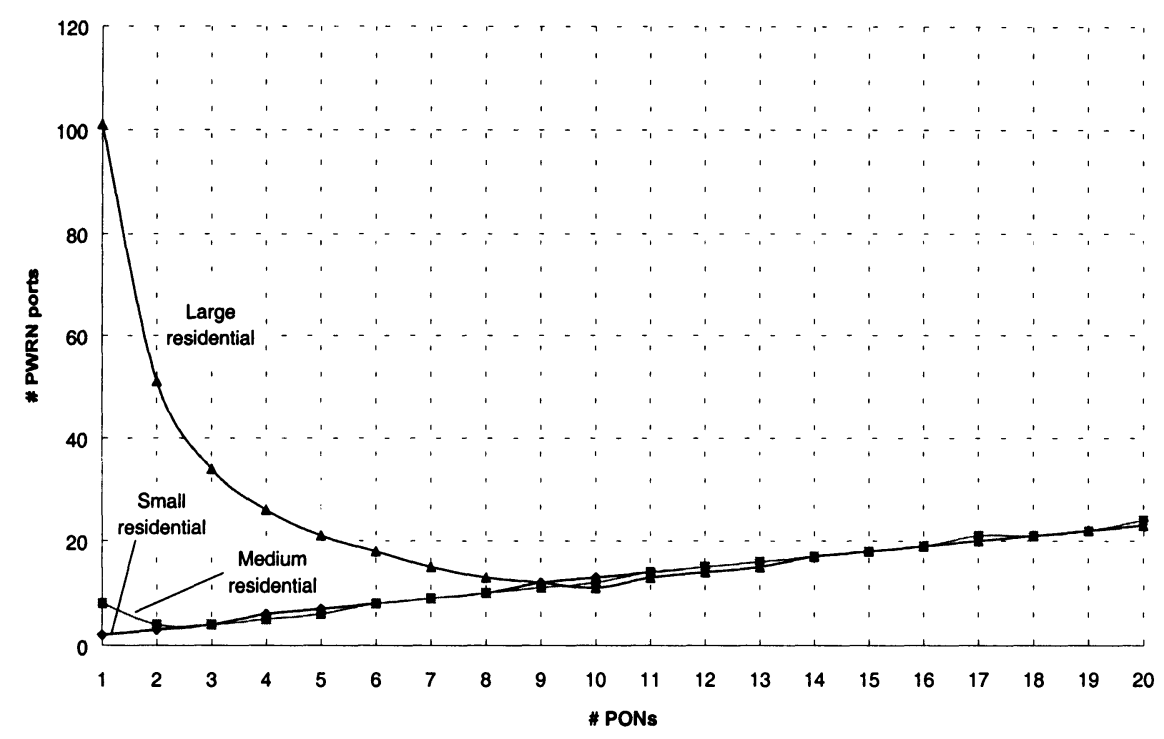

Figure 4. Total number of PWRN ports (Nt) vs. number of PONs (Nd) in case of residential traffic scenario (constraint: $\pi<10^{-5}$ )

In order to understand the real potentiality of the "switchless" network, in Figure 5, the number of users that can be supported by a single PWRN vs. the number of "dummy ports" is shown, considering the constraint $\pi<10^{-5}$. The data reported in this figure suggest that this kind of system could be used to serve a wide area, comparable with a medium-large country, with a lot of users.

\subsubsection{Comparison between analytical and simulation models}

In order to test the reliability of the analytical model, we have realised a simulative model, obtaining, for the same relevant cases, several results; such results have been compared with those arising by analytical model. The comparisons have shown that the difference among results obtained by analytical model (using Wilkinson and Fredericks model, considering the block at the output of "dummy ports"), and those obtained by simulation model is negligible. Moreover, it has been observed that the contribution of the block point at the output of "dummy ports" to the calculation of global blocking probability is negligible. On this basis, all the other results have been obtained neglecting the contribution of the block at the output of "dummy ports". 


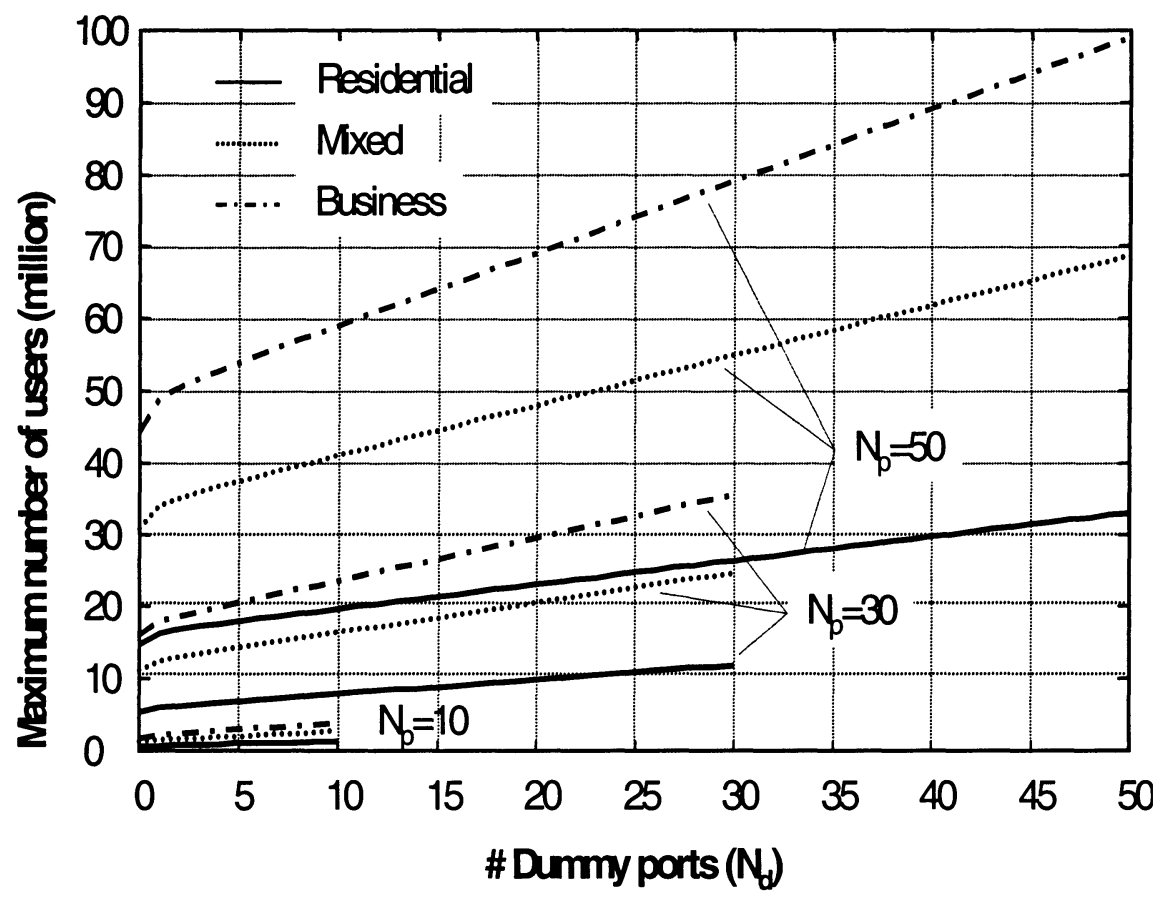

Figure 5. Maximum number of users vs. the number of dummy ports (Nd) for $\pi<10^{-5}$

\subsection{Analytical model - unbalanced traffic distribution}

Until now, it has been considered a uniform traffic distribution among PONs. Now, we remove this limitation and consider the more realistic case of an unbalanced traffic distribution: some PONs offer more traffic to the PWRN than others do. We assume that the total offered traffic by all PONs follows a gaussian distribution: there will be a single PON which offers, on average, more traffic than others PONs, with a certain standard deviation. Of course, on time, PON, which offers more traffic, will not be always the same, but we imagine a situation variable in time. Traffic type is still supposed only telephone. The analytical model is the same described in §2.1. There are only two differences: firstly, traffic parameters must be expressed as a sum of different terms:

$$
A_{o, t o t}=\sum_{i=1}^{N} A_{o}(i) \neq N^{*} A_{o}
$$


The second difference is that the requested number of "dummy ports" depends on PON activity: the PON which offers more traffic uses more "dummy ports" or wavelength converters; the PON which offers less traffic uses less "dummy ports".

\subsubsection{Network dimensioning results for unbalanced traffic distribution}

We have considered two possibility choices for network dimensioning: the first in which we choose a number of "dummy ports" equal to the maximum value requested by that PON which offers more traffic, and the number of wavelength converters is equal to the number of PONs. In the second we maintain constant the total available resources, increasing the number of "dummy ports", but reducing the number of wavelengths per "dummy port".

The major results obtained with the first network dimensioning type are shown below.

In Figure 6, assuming 10 input PONs belonging to the PWRN ports and a standard deviation equal to 2 , it is shown the blocking probability vs. the number of "dummy ports", for each PON; it is also shown, as comparison, the curve relative to the mean value of loss probability.

It can be seen that the PON that offers more traffic (in this graphic it is represented by curve labelled with the number 5) requires more "dummy ports" (offering more traffic to the PWRN, it needs more wavelength converters, that is, more auxiliary capacity), for a given blocking probability.

In Figure 7, it is shown the number of wavelength converters vs. the number of PONs, varying standard deviation; it is included also a curve for uniform traffic distribution. It can be seen that increasing the standard deviation value, system behaviour is close to system behaviour in uniform traffic case. Moreover, increasing number of PONs, number of wavelength converters decreases because, for a given total offered traffic at input of the system, traffic offered per each relation input PON i/output PON j decreases.

The major results obtained with the second dimensioning choice will be shown below.

It is worth to notice that in this case, considering that now the number of wavelength converters per "dummy port" is limited to a number less than the number of input PONs, we must use tunable wavelength converters in the recirculating lines. Besides, considering that the number of these devices are limited, another block point must be taken into account: infact, a call cannot be handled if there isn't a free wavelength converter in the re-circulating lines. Also this blocking probability is calculated by the Fredericks method. 


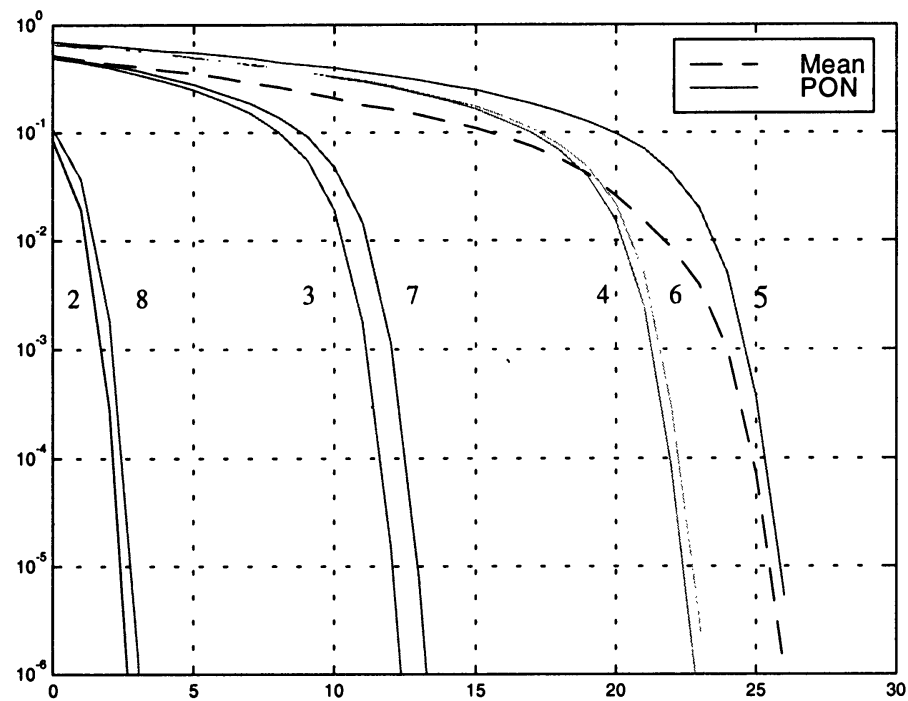

Figure 6. Blocking probability (ordinate) vs. the number of "dummy ports" (abscissa), for 10 PONs (each color is relative to a PON)

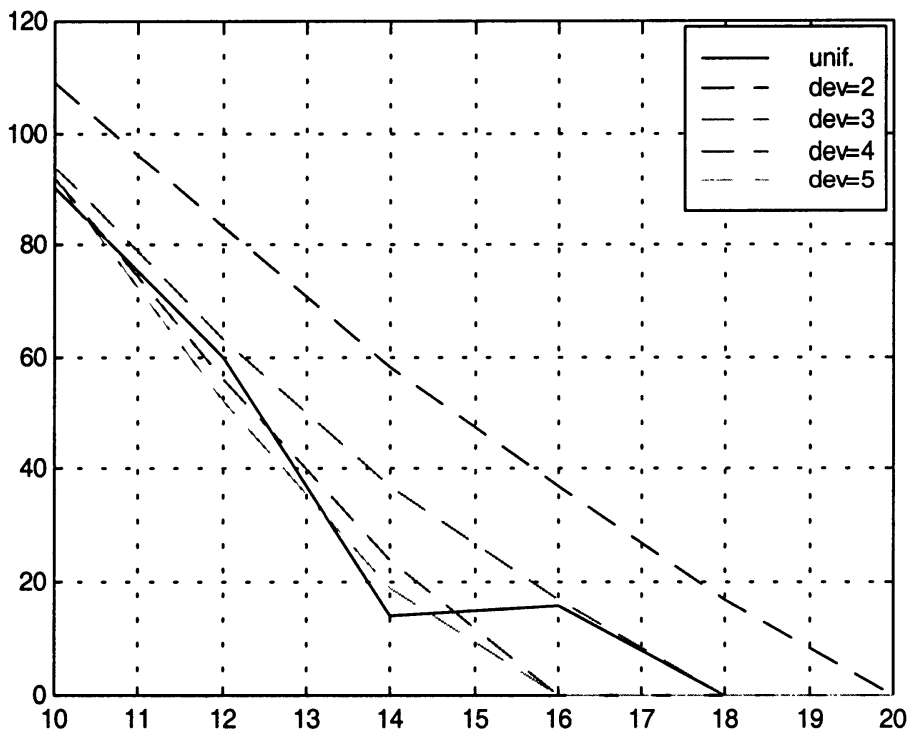

Figure 7. Number of wavelength converters (ordinate) vs. the number of PONs (abscissa), varying standard deviation 
In Figure 8, assuming a uniform traffic distribution among PONs, it is shown the blocking probability vs. the total number of wavelength converters, varying the number of wavelength converters per "dummy port" (that is, the number of wavelength converters effectively needed). It can see that, diminishing the number of wavelength converters per "dummy port", their total number decreases; however, this variation is not remarkable (for a blocking probability of $10^{-5}$, the number of wavelength converter change from 93, with $\mathrm{WC}=10$, to 70 , with $\mathrm{WC}=2$ ).

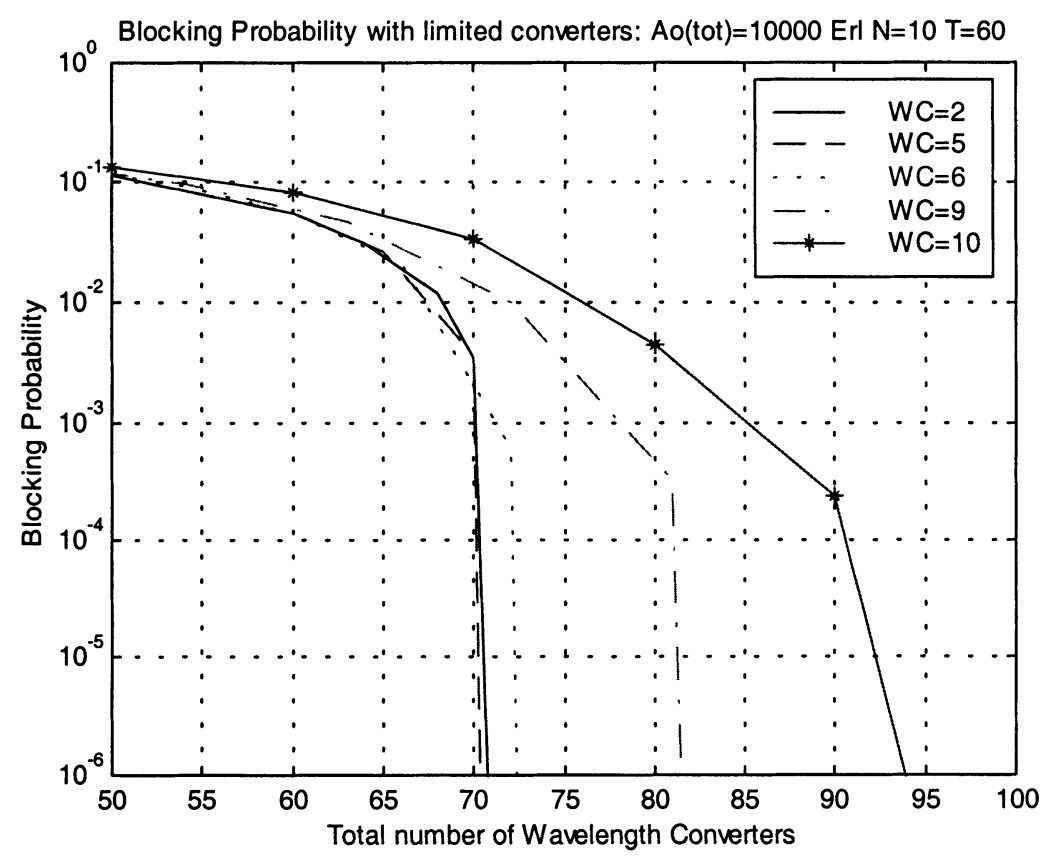

Figure 8. Blocking probability vs. the total number of wavelength converters, varying the number of wavelength converters (WC) per "dummy port", assuming a uniform traffic distribution among PONs

Different result is obtained considering an unbalanced traffic distribution among PONs, as shown in Figure 9: using five wavelength converters per "dummy port", the reduction in the total number of wavelength converters is very remarkable (from about 250 to 130 wavelength converters); it justify the higher cost and higher control complexity because of the use of tunable converters. 


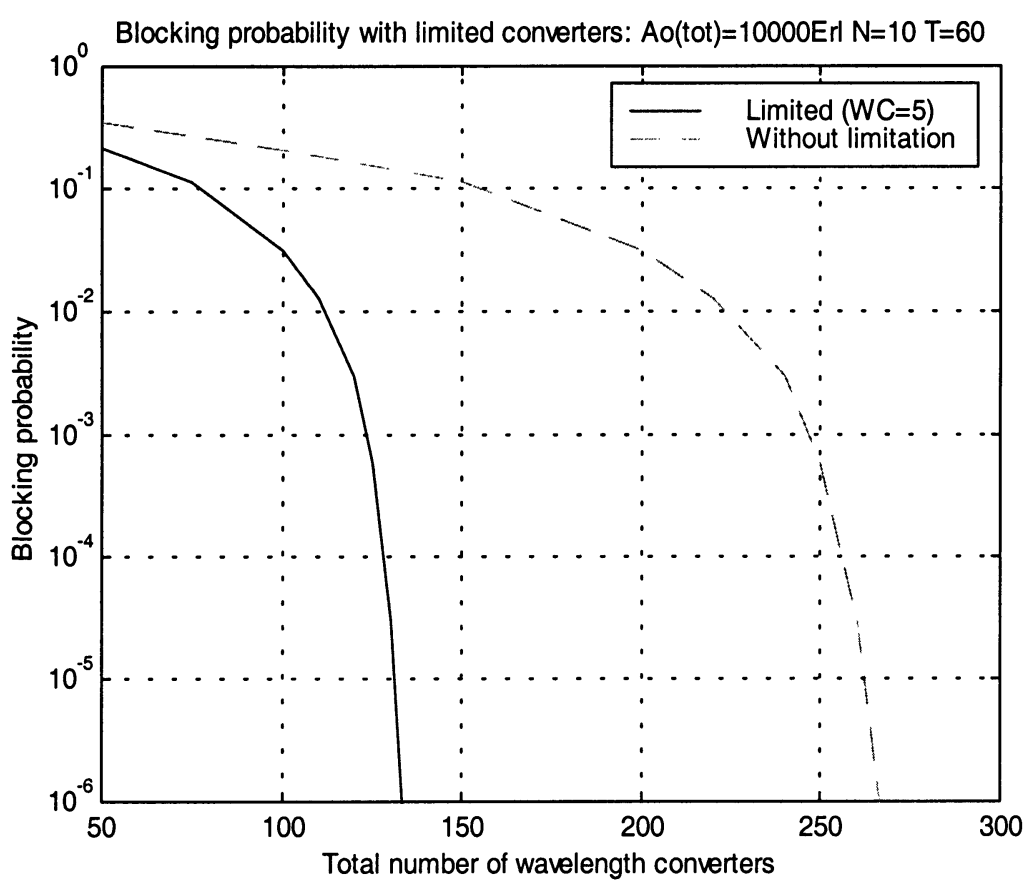

Figure 9. Blocking probability vs. the total number of wavelength converters, varying the number of wavelength converters per "dummy port", assuming an unbalanced traffic distribution among PONs

\subsection{Comparison between analytical and simulation models}

The validity of these models, under the hypotheses made, has been verified. The comparison, in the case of no limitations on wavelength converters, has shown that analytical and simulation models are very close; they are not coincident because, in simulation model, time -slots coincidence at the input and output of "dummy ports" have not been taken into account.

In case of limitation of wavelength converter per "dummy port", the validity of the models, and the hypotheses made have been verified through another simulation model. Also in this case, the comparison has shown that analytical and simulation models have a very close behaviour. 


\section{CONCLUSIONS}

In the framework of ACTS-SONATA project, it has been developed an analytical model that allows "switchless" network dimensioning, in the case of Poisson and Gaussian traffic, considering different user classes. These models have been demonstrated to be in full agreement with simulation models, also developed within the same project. The number of customers in a PON, the number of PONs, the number of re-circulating lines (i.e. the number of wavelength conversion blocks or "dummy ports"), and the total number of wavelength converters have been considered as design parameters. As results, the analyses reported for some relevant cases showed that is possible to assess the feasibility of a "switchless" network, since there are no constraints in realising networks covering even for large-scale areas.

On the basis of a gaussian traffic distribution, it has been seen that the PON which offers more traffic requires more "dummy ports"; moreover, the more unbalanced is the traffic offered, the higher is the number of "dummy ports" for a given blocking probability.

About network dimensioning, limiting wavelength converters number per "dummy port" and increasing these, we have found that advantages, in terms of reduction of total wavelength converter number, can be obtained in both cases of uniform and unbalanced traffic distribution: but, while in the first case the reduction of total converters is little, in presence of an unbalanced traffic distribution the advantages is higher, justifying the increment in cost and control complexity due to the use of tunable wavelength converters, on the re-circulating lines.

\section{BIBLIOGRAPHY}

[1] A. Hill, A. Carter, S. Armitage, J. Shabeer, M. Harmon, P. Rose, "A scalable and switchless network structure, employing $32 \times 32$ free-space grating multiplexer", IEEE Photonics Technol. Lett., vol.8, pp. 569-571, 1996.

[2] N. P. Caponio, A. M. Hill, R. Sabella, "Switchless Optical Network for Advanced Transport Architecture” LEOS'98, Paper WZ4, Orlando, Dec.1998, pp.358-359.

[3] T. Durhuus, et al., "All-optical wavelength conversion by semiconductor optical amplifiers", IEEE Journal of Lightwave Technology, vol. 14, no. 6, pp. 942-954, 1996.

[4] C. Joergensen, et al., "Wavelength conversion by optimized monolithic integrated MachZehnder interferometer ", IEEE Photonics Technology Letters, vol. 8, no. 4, pp. 521-524, 1996.

[5] W. Idler, et al., "10 Gb/s wavelength conversion with integrated multi-quantum well based 3 ports Mach-Zehnder interferometer", IEEE Photonics Technology Letters, vol. 8, no. 9, pp.1163-1165, 1996.

[6] J. Leuthold, et al., "All optical $2 \times 2$ switches with $20 \mathrm{~dB}$ extinction ratios", Electronics Letters, vol. 32, no. 24, November, pp. 2235-2236, 1996. 
[7] F. Ratovelomanana, et al., "Improvement of regeneration capabilities of an all optical wavelength converter, based on a Mach-Zehnder interferometer, by means of a phaseshifter section", IEE Electronics Letters, vol. 33, no. 19, pp. 1629-1630, 1997.

[8] K. Sato, Advances in Transport Network Technologies, Norwood, MA: Artech House, 1996.

[9] R. Sabella, P. Lugli, High Speed Optical Communications, chap. 12, Kluwer Academic Publishers, 1999.

[10] A.M. Hill, "WDM Networking in the Access Network and Beyond", ECOC'95 Optical Networking Forum Workshop, Paper S1.4, 1995.

[11] N. P. Caponio, A.M. Hill, F. Neri, R. Sabella, "Single-layer optical platform based on WDM/TDM multiple access for large-scale "switchless" networks", in press on European Transactions on Telecommunications, Special Issue on WDM Networks.

[12] J. Y. Hui, Switching and Traffic Theory for Integrated Broadband Networks, Kluwer Academy Press, 1990.

Stefano Binetti was born in Rome, Italy, in 1973. He received the degree in electronic engineering from University of Rome "Tor Vergata" in 1998. His research interests are in high-speed optical communications systems and WDM optical networks. In March 1998 he won a research fellowship and then, he joined CoRiTeL (Rome, Italy); he worked in the ACTS Project "SONATA". Now, he is with Pirelli Optical Systems where he is involved in a research \& development activity on optical networks. He is also following the standardisation activity on this topic.

Alessandro Bosco was born in Rome, Italy, on May 1973. He received the degree in telecommunication engineering from University of Rome "Tor Vergata" in 1999. His research interests are in High Speed Optical Systems, WDM optical networks and Physical Layer Modeling. In September 1999, he won a research fellowship and then, since September 1999, he joined CoRiTeL (Rome, Italy). Now he is working in the ACTS 351 Project named "SONATA".

Marco Listanti received his Dr. Eng. degree in electronics engineering from the University "La Sapienza" of Roma in 1980. He joined the Fondazione Ugo Bordoni in 1981, where has been leader of the group "TLC network architecture" until 1991. In November 1991 joined the INFOCOM Dept. of the University of Roma "La Sapienza", where he is Associate Professor in Switching Systems. Since 1994, he also collaborates with the Electronic Department of the University of Rome "Tor Vergata" where he holds courses in Telecommunication networks. He participated at several international research projects sponsored by EEC and ESA and is author of several papers published on the most important technical journals and conferences in the area of telecommunication networks. His current research interests focus on traffic control in IP networks and on the evolution of techniques for optical networking. Mr. Listanti has been representative of 
Italian PTT administration in international standardisation organisations (ITU, ETSI). Mr. Listanti is also a member of IEEE Communications and Computer Societies.

Roberto Sabella received the degree in electronics engineering (Laurea in Ingegneria Elettronica) from the University "La Sapienza" of Roma, Italy, in 1987. He then joined Ericsson Telecomunicazioni, Rome, Italy, where he was a Hardware Designer for about one-and-a-half years, and then as a Researcher on advanced fiber-optic communications. His research interests are in high-speed optical communication systems and optical networks. Since 1991, he led a group working on the modeling and simulation of advanced communication systems. He holds two patents on optical crossconnects, is co-author of a book on high-speed optical communications and author of more than 70 papers on scientific/technical journals and international conferences. Since May 1997, he has been with CoRiTel consortium as research technical co-ordinator. He has been Lecturer (professore a contratto) at the University of Rome "Tor Vergata" and at the Polytechnic of Bari, Italy. Mr. Sabella is member of the IEEE/LEOS Technical Committee on Optical Networks and Systems, and member of the editorial board of the new international journal Photonic Network Communications (New York: Kluwer Academic). For the same journal he has operated as guest editor for the special issues on WDM transport networks. Moreover, he has been invited to be one of the guest editors of a special issue on optical networks, for the journal Computer Networks (Elsevier). 